\title{
Thermochromic Infrared Metamaterials
}

\author{
Xinyu Liu* and Willie J. Padilla
}

The ability to control energy emitted from the surface of a material underpins many energy harvesting and thermal control applications ranging from thermophotovoltaics and low-E glass to spacecraft design and solar cells. However, there is little room for variability, since the total energy radiated from a blackbody is inexorably tied to its temperature - varying as the fourth power - as prescribed by the Stefan-Boltzmann (SB) law. ${ }^{[1]}$ Here, we demonstrate an artificial thermochromic material based on metamaterial micro-electro-mechanical systems (MEMS) which realize strong temperature dependent emission far exceeding the SB law over a band in the thermal infrared. Our device is fashioned to control its own temperature passively, i.e., at room temperature it exhibits low emissivities, but at a designed critical temperature, its emitted power density increases by over two orders of magnitude, thus cooling itself down. This "smart material" demonstrates a new direction for the realization of engineered thermal surfaces.

More than a hundred years before Planck placed blackbody radiation on firm theoretical ground with the dawn of quantum mechanics, Thomas Wedgwood - relative to Charles Darwin noticed that all objects in an oven, regardless of their chemical nature, size, or shape, became red at the same temperature. ${ }^{[2]}$ In time, the SB law would indeed verify Thomas' observation, thus unveiling a common property, and fundamental limitation, of most natural materials, i.e., their emission is solely determined by their temperature. There is, however, a class of materials which are capable of breaking the $\mathrm{T}^{4}$ dependence of the Stefan-Boltzmann law, known as thermochromic materials. More generally, chromogenics are a type of material that exhibit a change in optical properties - scattering, absorptivity, reflectivity, or transmissivity - as a result of an external stimulus. Some common examples are photochromic and electrochromic materials, where the external stimulus is light and electric field, respectively. ${ }^{[3]}$ Thermochromism, on the other hand, describes a material that changes its optical properties as a function of heat. ${ }^{[4]}$ Naturally occurring thermochromic materials have long been known ${ }^{[5]}$ and have been used for a handful of eccentric applications - mood rings and baby thermometers being wellknown examples. Within the last decade, however, alternative approaches involving composite materials have had a resurgence in study, due to design strategies involving the creation of thermochromic properties through the chemical or physical interaction of nonthermochromic components. ${ }^{[6]}$

Although some progress has been made utilizing the above described thermochromic materials, many applications are

X. Liu, Prof. W. J. Padilla

Department of Electrical and Computer Engineering

Duke University

Durham, NC 27708, USA

E-mail: xinyu.liu1@duke.edu

DOI: 10.1002/adma.201504525 still limited by the Stefan-Boltzmann law. Electromagnetic metamaterials are engineered composites that can potentially overcome the $\mathrm{T}^{4}$ dependence of the SB law, since the optical properties are obtained from the geometry of the constituent components, rather than directly from their chemical composition. Therefore, the temperature dependent spectral emissivity of metamaterial surfaces may be tailored and controlled via the geometry of the structures. Although tunable metamaterials have made significant progress in achieving real-time control of electromagnetic waves, in optical, ${ }^{[7,8]}$ infrared, ${ }^{[9-11]}$ $\mathrm{THz},{ }^{[12-15]}$ and lower frequencies, ${ }^{[16,17]}$ there has yet to emerge a viable solution to directly control the temperature dependence of surface radiation. Here we propose and demonstrate an artificial thermochromic material, fashioned from a metamaterial emitter ${ }^{[18]}$ combined with bimaterial MEMS, (Figure 1), which is capable of achieving certain desired thermally dependent surface emission.

The metamaterial consists of a metallic ground plane, a dielectric layer, and a top patterned metallic layer, (see Figure 1), and has been shown to yield wavelength dependent spectral emissivity, with peak values approaching the ideal blackbody maximum. ${ }^{[18]}$ The high absorption and emission values arise from the confluence of magnetic and electric resonances, each similar to a Lorentz oscillator. Thus, if either of the resonances is changed in amplitude, center frequency, or scattering frequency, the coupling between these two resonances may deviate from optimal, thus realizing values decreasing from unity. We have designed our MEMS metamaterial (see the Experimental Section) to attain relatively low emissivities at room temperature. In this configuration, the top metamaterial pattern is suspended above the ground plane (Figure 1a) and designed to have non-optimal coupling to the absorptive resonance. Bimaterial arms, fashioned from gold and silicon nitride, support the top metamaterial layer and - when heat is applied - expand and bend down due to the difference between the thermal expansion coefficients. As a result, the distance between the suspended metamaterial layer and the ground plane is reduced. When the top metamaterial layer comes in contact with the ground plane (Figure 1b) a state of high infrared emission is achieved (see Section 2 of the Supporting Information for more detail). Our artificial thermochromic material thus follows a super Stefan-Boltzmann curve and realizes a monotonically increasing temperature dependent spectral emissivity. In contrast, most materials realize only modest wavelength dependent emissivities and, more importantly, little or no temperature dependent emissivity - only varying as the fourth power of temperature as described by the SB law.

The experimental wavelength dependent spectral emissivity is shown in Figure 2a for a number of different geometrical configurations of our metamaterial, as determined by temperature. The metamaterial layer is suspended in the room temperature (nonoptimized) state and achieves a peak spectral 
a

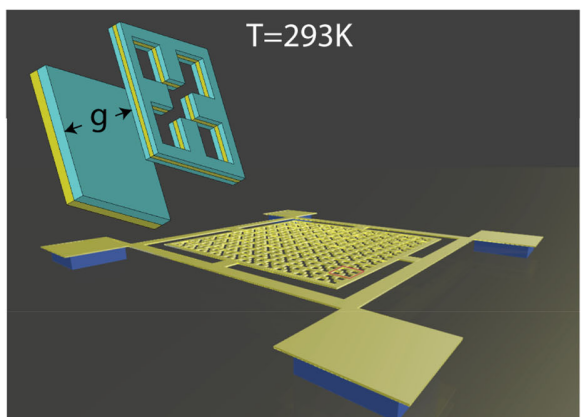

b

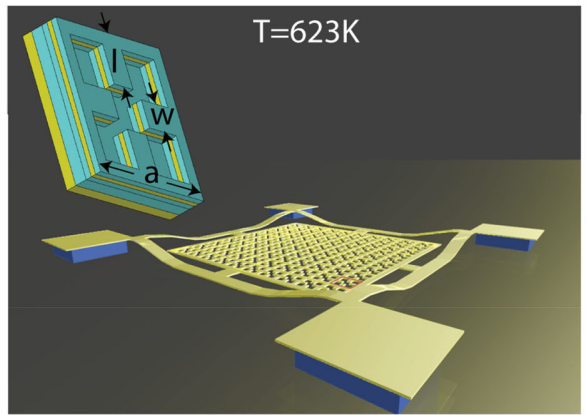

C

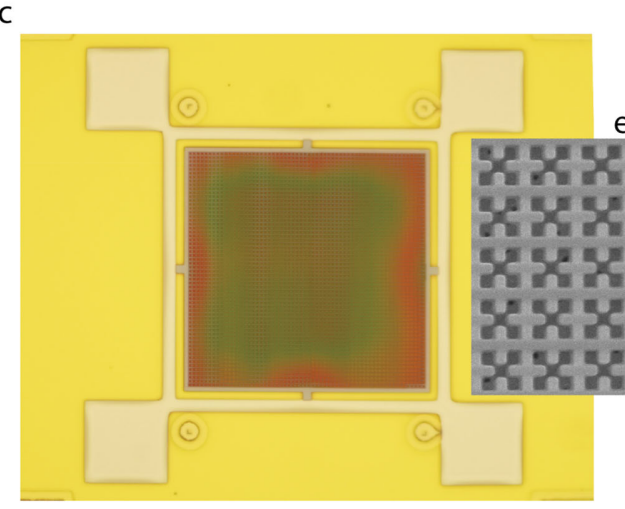

d

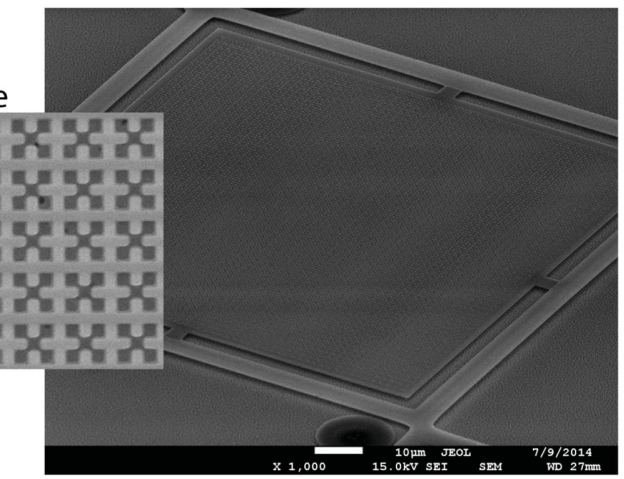

Figure 1. Geometrical design and images of the thermochromic metamaterial. a,b) Schematic of the device and the unit cell dimensions (insets in plot $\mathrm{a}$ and b) when operated in the suspended (a) and down (b) states. $a=1.8 \mu \mathrm{m}, g=1.6 \mu \mathrm{m}, w=0.33 \mu \mathrm{m}, I=0.64 \mu \mathrm{m}$. c) Optical picture of the fabricated metamaterial. d) scanning electron microscope (SEM) picture of the metamaterial at a viewing angle of $60^{\circ}$ from the surface normal. e) SEM image of the suspended metamaterial layer.

emissivity of just over $50 \%$ (solid blue curve). As temperature is elevated, the metamaterial approaches the surface and the spectral emissivity monotonically increases (gray curves). At the maximum temperature characterized $(623 \mathrm{~K})$, the peak spectral emissivity achieves a value of $81 \%$ at a wavelength of $5.2 \mu \mathrm{m}$ - red curve in Figure 2a (see Section 2 of the Supporting Information). We plot the differential emissivity $(D E)$, defined as $D E(\lambda, T)=E(\lambda, T)-E(\lambda, 293 \mathrm{~K})$, and peak differential emissivity in Figure $2 c, d$, respectively, for various temperatures. It can be observed that there is a marked increase in the rate of emission from the metamaterial, beginning at a temperature of $\approx 473 \mathrm{~K}$, whereupon the metamaterial makes contact with the ground plane. We perform finite difference time domain (FDTD) simulations of the thermochromic metamaterial and find relatively good agreement with experiment, see Figure $2 \mathrm{~b}$, thus confirming our experimental observations.

In order to understand the particular temperature dependence of the spectral emissivity, we perform hyperspectral imaging over the surface of our thermochromic metamaterial. In Figure 3a,b, we show false color plots of the emissivity of the metamaterial at 293 and $623 \mathrm{~K}$, as well as the differential emissivity in Figure 3c. Figure 3a demonstrates that the metamaterial achieves an emissivity of approximately $50 \%$ at room temperature, and Figure $3 \mathrm{~b}$ at $623 \mathrm{~K}$ values of $80 \%$ are reached. It can be observed, however, that there is some nonuniformity in the spectral emissivity of the metamaterial - most likely the result of a slight stress-induced curvature of the metamaterial surface, ${ }^{[19]}$ and this nonuniformity may also be responsible for the form of the temperature dependence emissivity shown in Figure $2 \mathrm{~d}$.

We next perform a calculation in order to determine the temperature dependent radiative properties of our thermochromic metamaterial. The spectral radiance of our device can be derived from the spectral emissivity by multiplying with the appropriate blackbody radiation spectrum for a given temperature. ${ }^{[20]}$ Figure $4 \mathrm{a}$ shows the spectral radiant power density of our sample for a number of different temperatures ranging from 293 to $623 \mathrm{~K}$. In order to compare the temperature dependence of our metamaterial to that of a blackbody, we integrate the spectral radiant power density of both our sample and that of a blackbody from 3 to $8 \mu \mathrm{m}$, i.e., the measured spectral range (see Section 4 of the Supporting Information). In Figure $4 \mathrm{~b}$, we plot the temperature dependence of the integrated radiant power densities (normalized with respect to room temperature) of both a blackbody (open black circles) and our sample (open red circles). As can be observed from Figure $4 \mathrm{~b}$ - left axis - the integrated radiative power density of the thermochromic metamaterial at $623 \mathrm{~K}$ is 215 times greater than that at room temperature, while the blackbody only increases by a factor of 90 .

The Stephan-Boltzmann law dictates that the total power per unit area emitted at all frequencies - termed the radiant exitance $(M)$ - by a surface is proportional to the fourth power of its absolute temperature and may be expressed as 

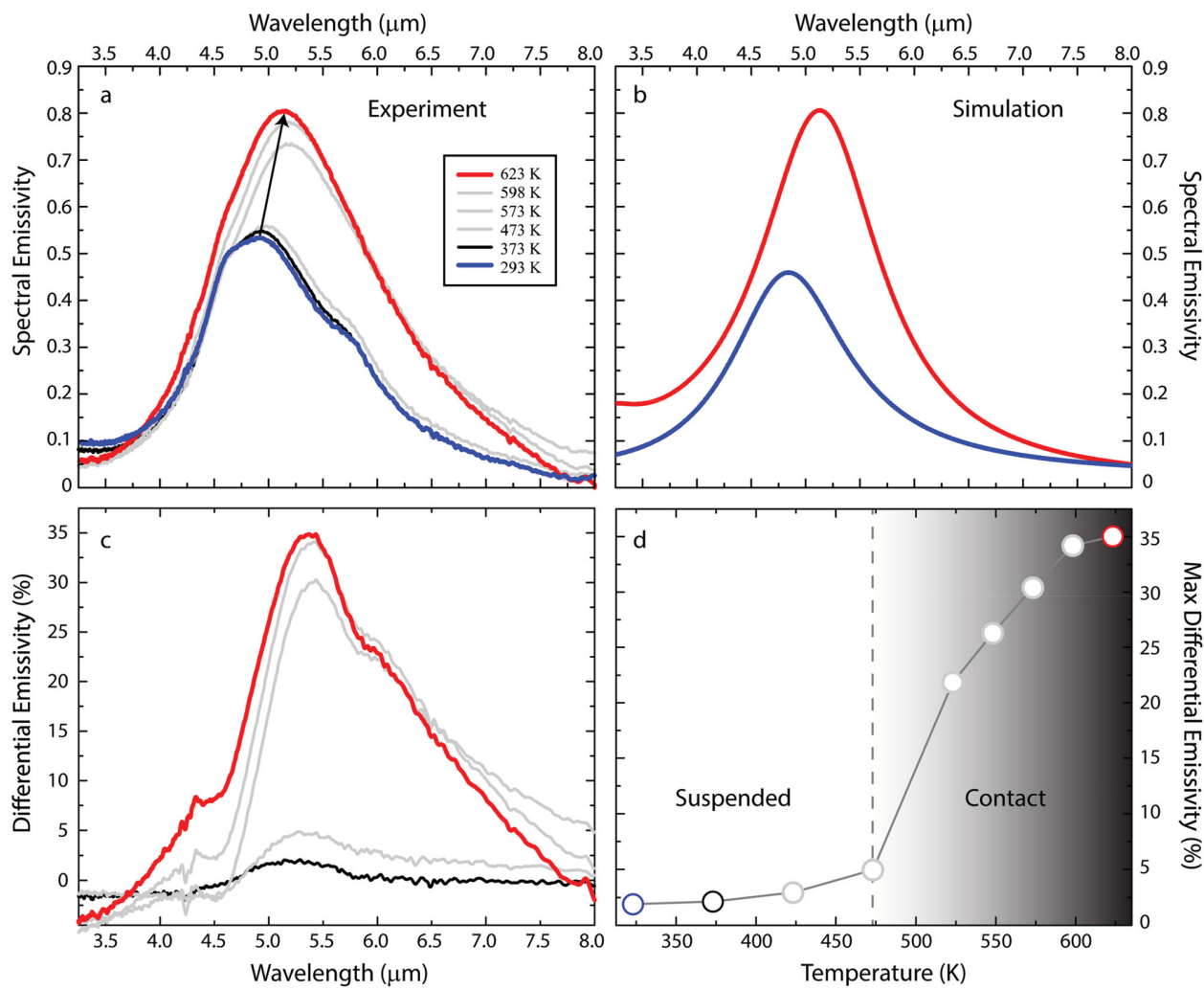

Figure 2. Simulated and measured thermochromic metamaterial emission. a) Experimental characterization of the wavelength dependence of the emissivity shown for various temperatures. The blue and red curves are for temperatures of 293 and $623 \mathrm{~K}$, respectively. b) Simulated room temperature and $623 \mathrm{~K}$ emissivity. c) Wavelength dependent differential emissivity as defined in the text. d) Maximum differential emissivity as a function of temperature. Dashed vertical line separates regions when the metamaterial layer is suspended compared to when it begins to contact the bottom ground plane.

$M(T)=\varepsilon \sigma T^{4}$

where $\sigma=5.67 \mathrm{e}^{-8}\left(\mathrm{~W} \mathrm{~m}^{-2} \mathrm{~K}^{-4}\right)$ is the Stefan-Boltzmann constant and $\varepsilon$ is the emissivity ( $\varepsilon=1$ for a blackbody). In order to explore and compare $M(T)$ for our thermochromic metamaterial to that of an idealized blackbody, we must extend our data over all wavelengths. We thus fit the experimental spectral emissivity with Lorentz model and calculate the temperature dependent radiant exitance using (see Section 4 of the Supporting Information for more detail)

Emissivity

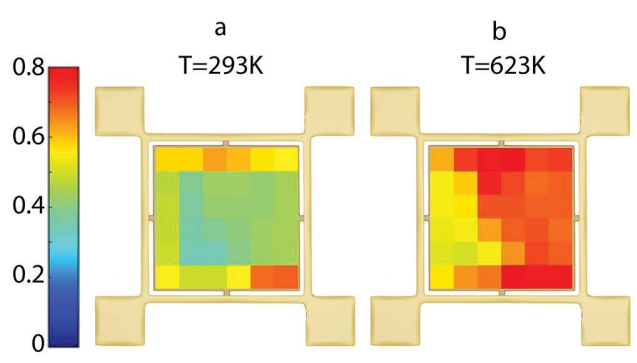

$M_{\mathrm{m}}(T) \equiv \int_{0}^{\infty} E(\lambda, T) \frac{2 \pi h c^{2}}{\lambda^{5}} \frac{1}{e^{h c / \lambda k_{\mathrm{B}} T}-1} \mathrm{~d} \lambda$

The open gray squares shown in Figure $4 \mathrm{~b}$ reveal the temperature dependence of $M_{\mathrm{m}}(T)$ from Equation (2). In order to directly compare the temperature dependence of our metamaterial to that of a blackbody, we fit with a power law equation expressed as $M_{\mathrm{m}}(T)=a T^{b}$, where $a$ and $b$ are constants. Strikingly, we find a best fit exponent value of $b=6.2$, thus indicating that our thermochromic metamaterial provides a higher

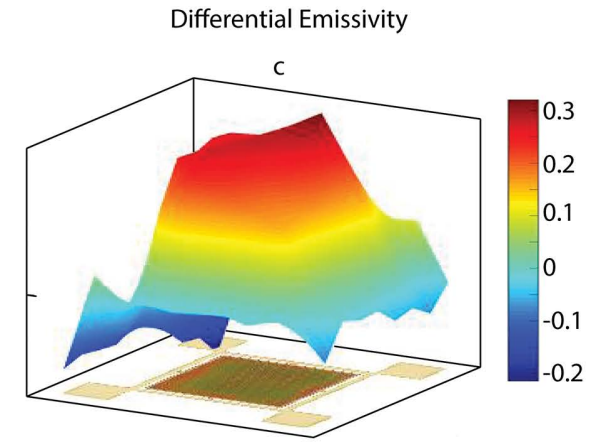

Figure 3. Measured spatial dependence of the emissivity and differential emissivity across the surface of the thermochromic metamaterial. a) Color map of the absorption at room temperature and b) $623 \mathrm{~K}$ (shown over an optical image of the fabricated metamaterial MEMS), both at a wavelength of $5.2 \mu \mathrm{m}$. c) Isometric view of the spatial dependence of the differential emission at a wavelength of $5.2 \mu \mathrm{m}$ (defined in the text) with the colormap shown to the right. 
a

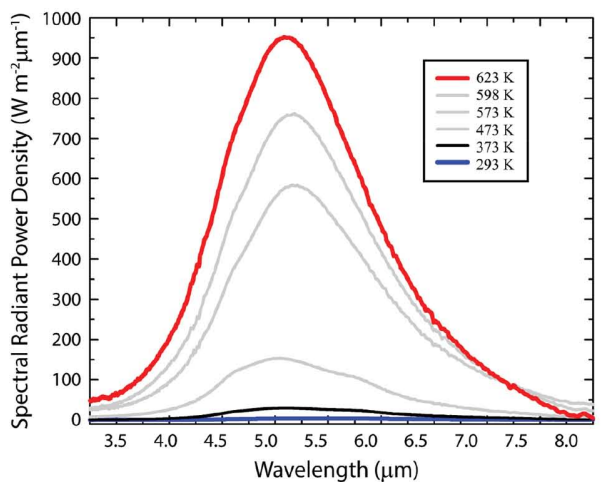

b

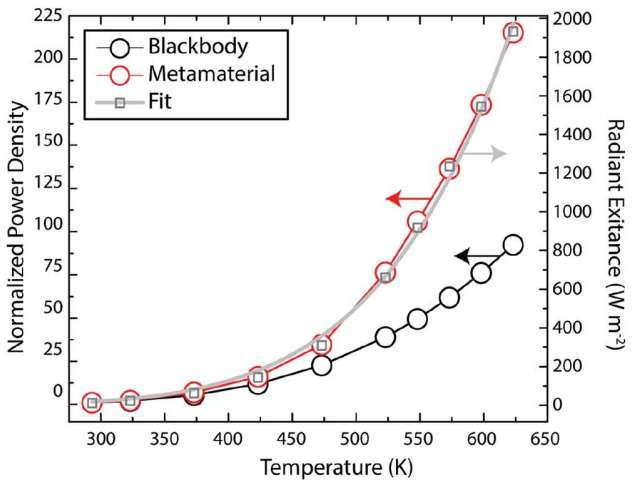

Figure 4. Emitted power density of the thermochromic metamaterial. a) Power density per wavelength for different temperatures as indicated in the inset legend. b) Normalized power density (left vertical axis) as a function of temperature (defined in the Supporting Information) for both the metamaterial (open red circles) and blackbody (open black circles). The open gray squares show the radiant exitance (right vertical axis) determined by a Lorentz model fit to the experimental spectral emissivity data (see Section 4 of the Supporting Information for more information). The solid gray curve is a power law fit to the open gray squares.

power law relation than that possible with natural materials which follow Equation (1).

We have demonstrated that metamaterials may function as artificial thermochromic materials providing emissive properties that exceed what is possible with other systems which typically follow the Stephen-Boltzmann law. Although there have been a few demonstrations of dynamic control of thermal radiation using natural thermochromic materials, ${ }^{[21,22]}$ this type of thermal tuning relies on a phase transition and is therefore difficult or impossible to extend to different temperatures and/or alternative bands of the electromagnetic spectrum. In contrast, through judicious geometrical design metamaterials permit an independent tuning of the resonance wavelength and transition temperature. Thus the working temperature and operational band of our artificial thermochromic device can potentially cover a significant portion of the wavelength-temperature phase space.

In conclusion, we have proposed, fabricated and characterized a new artificial thermochromic material which achieves outstanding thermal emission performance beyond what is predicted by the Stefan-Boltzmann law. The emissivity of our device is a monotonically increasing function of temperature and reaches a peak emissivity of $81 \%$ at $623 \mathrm{~K}$. Our results verify the potential of metamaterials to function as smart materials, i.e., able to passively control their own temperature, which may be useful in many fields such as solar energy harvesting and space exploration. Our work is general and not limited to the case experimentally demonstrated, but our procedure may be replicated to achieve temperature dependent emissivity in other desired temperature and frequency regions. Metamaterials permit fabrication of advanced engineered surfaces capable of independent control of the peak emission wavelength and the temperature dependence of the emissivity.

\section{Experimental Section}

Simulation: Simulations were performed using the FDTD solver in computer simulation technology (CST) 2014 microwave studio.
Sample Fabrication and Characterization: The sample fabrication started with a $120 \mathrm{~nm}$ gold film deposition. Then, a $140 \mathrm{~nm}$ silicon nitride film was deposited by plasma enhanced chemical vapor deposition (PECVD), followed by spin coating a $1.6 \mu \mathrm{m}$ SU-8 sacrificial layer. A $70 \mathrm{~nm}$ thick silicon nitride layer was coated by PECVD and $55 \mathrm{~nm}$ gold was patterned by standard E-beam lithography process. A $70 \mathrm{~nm}$ silicon nitride was deposited again and a $60 \mathrm{~nm}$ titanium mask, which has the same pattern as the gold layer, was coated by E-beam lithography with an alignment process. The silicon nitride was removed (patterned) by reactive ion etching (RIE), followed by removal of the titanium mask. Finally, the device was released by plasma etching the SU-8 sacrificial layer. Optical and SEM pictures of the fabricated sample are shown in Figure 1c,d (The fabrication flow is shown in Figure S1 in the Supporting Information).

The artificial thermochromic material consists of five sub-wavelength thick layers - schematic of a single unit cell is shown in the insets to Figure 1a,b. Three of the layers form a suspended structure and consist of a silicon nitride - gold - silicon nitride sandwich. This symmetrical three-layered design was utilized to minimize undesirable bending during the application of heat, ${ }^{[7]}$ and was lithographically patterned into a Babinet metamaterial comprised of $60 \times 60$ unit cells. The other two layers consist of a silicon nitride layer lying above a gold ground plane. The suspended metamaterial layer is square and attached to the midpoints of bimaterial beams, lying around the perimeter, which were then connected to anchor blocks (blue colors) lying at each corner as shown in Figure 1.

The sample was mounted on a thermal stage and characterized with an infrared microscope coupled to a Fourier transform infrared (FTIR) spectrometer. The wavelength dependent reflectivity $(R)$ of the sample was measured from room temperature to just over $620 \mathrm{~K}$, as shown in Figure 2a. The bimaterial beams supported the free-standing metamaterial absorber top layer and were fashioned to expand as a function of increasing temperature. The suspended metamaterial layer has a slight bend and thus at temperatures above $473 \mathrm{~K}$, the contact area expanded until the maximum temperature of $623 \mathrm{~K}$ was reached. With the increase in temperature, the reflectivity minimum decreased continuously from $47 \%$ to $20 \%$ (the measurement errors are within $4 \%$ ), while the position, shifted from 4.9 to $5.2 \mu \mathrm{m}$.

\section{Supporting Information}

Supporting Information is available from the Wiley Online Library or from the author. 


\section{Acknowledgements}

The research presented in this work was supported through funding from the Department of Energy under contract number DE-SC0014372.

Received: September 14, 2015 Revised: October 15, 2015

Published online: November 30, 2015

[1] L. Boltzmann, Ann. Phys. 1884, 258, 291.

[2] R. A. Serway, C. J. Moses, C. A. Moyer, Modern Physics, Brooks/Cole, Belmont, CA, USA 2004

[3] P. Kiria, G. Hyettb, R. Binionsa, Adv. Mater. Lett. 2010, 1, 86.

[4] The term thermotropic is sometimes used to further clarify the exact optical property that is changing.

[5] G. Mastrotheodoros, K. Beltsios, N. Zacharias, Mediterranean Archaeol. Archaeometry 2010, 10, 37.

[6] A. Seeboth, D. Lotzsch, R. Ruhmann, O. Muehling, Chem. Rev. 2014, 114, 3037.

[7] J. Y. Ou, E. Plum, L. Jiang, N. I. Zheludev, Nano Lett. 2011, 11, 2142.

[8] Q. Liu, Y. Cui, D. Gardner, X. Li, S. He, I. I. Smalyukh, Nano Lett. 2010, 10, 1347.

[9] T. Driscoll, S. Palit, M. M. Qazilbash, M. Brehm, F. Keilmann, B. G. Chae, S. J. Yun, H. T. Kim, S. Y. Cho, N. M. Jokerst, D. R. Smith, D. N. Basov, Appl. Phys. Lett. 2008, 93, 024101.
[10] M. J. Dicken, K. Aydin, I. M. Pryce, L. A. Sweatlock, E. M. Boyd, S. Walavalkar, J. Ma, H. A. Atwater, Opt. Express 2009, 17, 18330

[11] X. L. Liu, W. J. Padilla, Adv. Opt. Mater. 2013, 1, 559.

[12] H. T. Chen, W. J. Padilla, J. M. O. Zide, A. C. Gossard, A. J. Taylor, R. D. Averitt, Nature 2006, 444, 597.

[13] H. T. Chen, J. F. O'Hara, A. K. Azad, A. J. Taylor, R. D. Averitt, D. B. Shrekenhamer, W. J. Padilla, Nat. Photonics 2008, 2, 295.

[14] W. M. Zhu, A. Q. Liu, X. M. Zhang, D. P. Tsai, T. Bourouina, J. H. Teng, X. H. Zhang, H. C. Guo, H. Tanoto, T. Mei, G. Q. Lo, D. L. Kwong, Adv. Mater. 2011, 23, 1792.

[15] D. Shrekenhamer, W. C. Chen, W. J. Padilla, Phys. Rev. Lett. 2013, 110, 177403.

[16] I. Gil, J. Bonache, J. Garcia-Garcia, F. Martin, IEEE Trans. Microwave Theory Tech. 2006, 54, 2665.

[17] L. Kang, Q. Zhao, H. J. Zhao, J. Zhou, Opt. Express 2008, 16, 8825.

[18] X. L. Liu, T. Tyler, T. Starr, A. F. Starr, N. M. Jokerst, W. J. Padilla, Phys. Rev. Lett. 2011, 107, 045901.

[19] W. D. Cowan, V. M. Bright, A. A. Elvin, D. A. Koester, Proc. SPIE 1997, 3225, 56.

[20] M. Diem, T. Koschny, C. M. Soukoulis, Phys. Rev. B 2009, 79, 033101.

[21] M. A. Kats, D. Sharma, J. Lin, P. Genevet, R. Blanchard, Z. Yang, M. M. Qazilbash, D. N. Basov, S. Ramanathan, F. Capasso, Appl. Phys. Lett. 2012, 101, 221101.

[22] M. A. Kats, R. Blanchard, S. Y. Zhang, P. Genevet, C. H. Ko, S. Ramanathan, F. Capasso, Phys. Rev. X 2013, 3, 041004. 